\title{
Free-Space Fiber-Optic Switches Based on MEMS Vertical Torsion Mirrors
}

\author{
Shi-Sheng Lee, Member, IEEE, Long-Sun Huang, Chang-Jin Kim, and Ming C. Wu, Member, IEEE
}

\begin{abstract}
This paper reports on the design, fabrication, and performance of a novel MEMS (micro-electro-mechanicalsystem) fiber-optic switch based on surface-micromachined vertical torsion mirrors. The vertical torsion mirror itself can be used as a $1 \times 2$ or an $\mathrm{ON}-\mathrm{OFF}$ switch. A $2 \times 2$ MEMS fiber-optic switch with four vertical torsion mirrors has also been fabricated. The switching voltage is measured to be $80 \mathrm{~V}$ for switching angles of $45^{\circ}$. We have achieved a switching time of less than $400 \mu \mathrm{s}$ (fall time) and an optical insertion loss of 1.25 dB for single-mode fibers. In addition, a bulk-micromachined silicon submount has been developed to package the switch with microball lenses and multimode fibers with passive alignment. With the micromachined switch chip and the hybrid-packaging scheme, the size, weight, and potentially the cost of the fiber-optic switches can be dramatically reduced.
\end{abstract}

Index Terms - Fiber data distribution interface (FDDI), microactuators, micro-electro-mechanical devices, optical fiber switches.

\section{INTRODUCTION}

$\mathbf{T}$ HERE has been a significant growth of optical fiber networks. Optical fiber offers many advantages compared with electric cables, including high bandwidth, low-loss, lightweight, immunity from electromagnetic interference, and the ability to increase the network capacity by wavelength division multiplexing (WDM). The rapid growth of optical fiber communication networks has created a large demand for many optical components, including optical switches. Low-cost optical components are particularly important for fiber-based local area networks (LAN).

Fiber-optic switches are used to reconfigure the network and/or increase its reliability. For example, the FDDI (fiber data distribution interface) fiber-optic ring network employs optional $2 \times 2$ fiber-optic switches, called optical bypass switches, to bypass the failed nodes. Low optical insertion loss and low crosstalk are two of the most important requirements for network optical switches. Generally speaking, optomechanical switches provide the lowest insertion loss and crosstalk among all optical switches. However, conventional optomechanical switches are bulky, slow, and expensive.

Recent advances in micro-electro-mechanical systems (MEMS) have opened up many new possibilities for

Manuscript received October 16, 1998. This work was supported by DARPA and the Packard Foundation.

S.-S. Lee and M. C. Wu are with the Department of Electrical Engineering, University of California at Los Angeles, Los Angeles, CA 90095 USA (email:wu@ee.ucla.edu).

L.-S. Huang and C.-J. Kim are with the Department of Mechanical and Aerospace Engineering, University of California at Los Angeles, Los Angeles, CA 90095 USA.

Publisher Item Identifier S 0733-8724(99)00516-2. optical and optoelectronic systems. Movable structures, microactuators, micropositioners, and micro-optical elements can be monolithically integrated on the same substrate using batch-processing techniques. Recently, there has been a growing interest in applying the MEMS technology to improve the performance and reduce the cost of optomechanical switches [1]-[6]. Bulk-micromachining of silicon has been combined with external actuators to implement $2 \times 2$ switches [1]. A monolithic $2 \times 2$ matrix switch has also been demonstrated by bulk micromachining [2]. Deep reactive ion etching has been employed to fabricate $2 \times 2$ switches on silicon-on-insulator (SOI) wafers [3]. However, most of these approaches require special fabrication processes.

Surface-micromachining technique based on standard processes, on the other hand, offers greater flexibility and shorter development cycles. The surface-micromachining process is particularly suitable for implementing free-space integrated optics. It has been shown that three-dimensional microoptical elements, micropositioners, and microactuators can be simultaneously fabricated by the same surface-micromachining process [7]-[9]. The micro-optical elements can be optically prealigned in the design stage to within the microfabrication tolerance. Previously, we have used the surfacemicromachining technique to demonstrate a fully actuated $2 \times 2$ fiber-optic switch [10]. A fall time of $15 \mathrm{~ms}$ and a rise time of $6 \mathrm{~ms}$ have been achieved. However, optical switches with faster response time are desired for most applications.

In this paper, we report on a novel $1 \times 2$ fiber-optic switch based on surface-micromachined vertical torsion mirrors. The vertical torsion switches have submillisecond switching time and better reliability. A $2 \times 2$ fiber-optic switch comprising multiple vertical torsion mirror elements is also demonstrated. Optical insertion loss of $1.25 \mathrm{~dB}$ has been obtained for single-mode fibers. .In addition, a new hybrid packaging scheme has been developed using bulk-micromachined silicon submount for low cost manufacturing of the switches. With the monolithic design, the batch fabrication process, and the hybrid packaging scheme, the size, weight, and potentially the cost of the fiber-optic switches can be dramatically reduced.

\section{DESIGN AND FABRICATION OF THE VERTICAL TORSION MIRROR}

The schematic drawing of the vertical torsion mirror switch is shown in Fig. 1. It consists of a vertical torsion mirror and a back electrode. The angle between the vertical torsion mirror and the back electrode is designed to be $45^{\circ}$. Both the vertical torsion mirror and the back electrode are realized by the microhinge technology [11]. The switch is fabricated by 


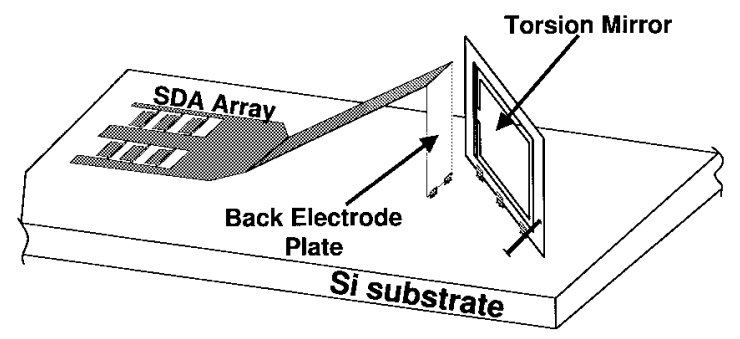

Fig. 1. Schematic drawing of the vertical torsion mirror switch.

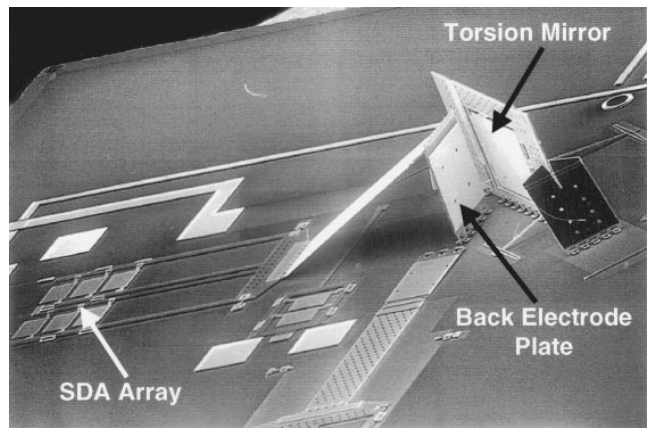

Fig. 2. Scanning electron micrograph (SEM) of the vertical torsion mirror switch.

the three-layer polysilicon surface-micromachining technology at MEMS Technology Application Center at North Carolina (MCNC) under the DARPA supported Multi-User MEMS Processes (MUMP's) service. Fig. 2 shows the scanning electron micrograph (SEM) of the switch. The back electrode plate is integrated with a scratch drive actuator (SDA) [12] array for self-assembly. The self-assembly approach dramatically reduces the assembly time of the switch. This is particularly important for the more sophisticated switches that employ multiple vertical torsion mirrors. The self-assembly structure is not included in the current mirror structure due to the constraint in device area. The operating principle of the switch is illustrated in Fig. 3. Without bias, the light from the input fiber is reflected by the $45^{\circ}$ mirror to fiber B [Fig. 3(a)]. When a voltage is applied to the switch, the mirror is moved out of the optical path and the input light is directly transmitted to fiber C [Fig. 3(b)].

\section{A. Vertical Torsion Mirror}

The vertical torsion mirror is mounted in a fixed polysilicon frame, as shown by the SEM in Fig. 4. The mirror is connected to the frame through a pair of $2-\mu \mathrm{m}$-wide, $82-\mu \mathrm{m}$-long and $0.55-\mu \mathrm{m}$-thick torsion beams. A $5-\mu \mathrm{m}$-wide and $2-\mu \mathrm{m}$-thick polysilicon stopper beam is integrated with the supporting frame to prevent overshoot of the mirror plate when the bias is released. The mirror plate is $200 \mu \mathrm{m}$ wide, $160 \mu \mathrm{m}$ tall and $1.5 \mu \mathrm{m}$ thick. The surface of the mirror is coated with $0.1-\mu \mathrm{m}$-thick gold to improve its reflectivity. The microhinge allows the supporting frame to rotate out of the Si surface and become perpendicular to the substrate. The frame is fixed by two side support plates and a pair of spring latches. The two spring latches are anchored to the opposite sides of the frame to reduce the tilt angle of the mirror plate and to

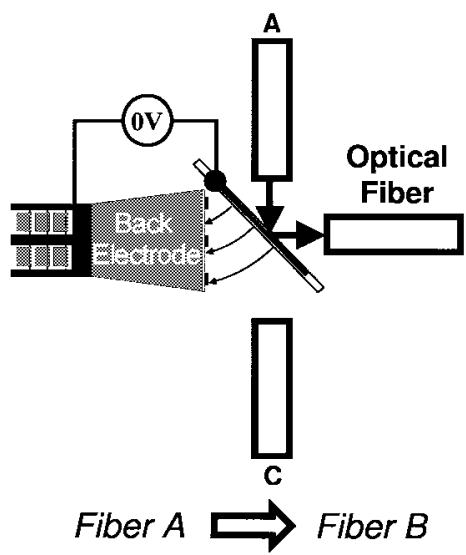

(a)

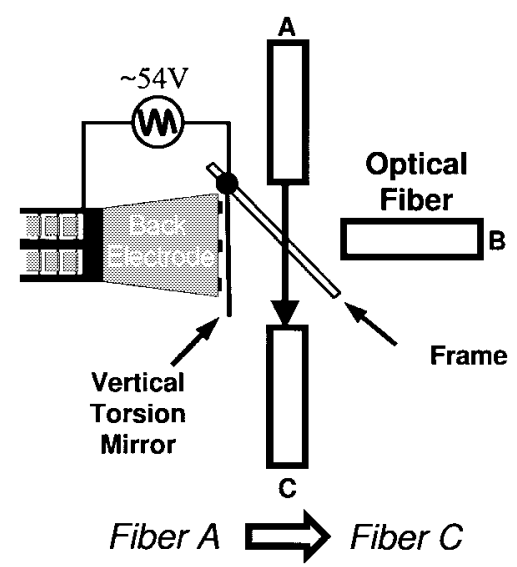

(b)

Fig. 3. Schematic illustration of operation principle of $1 \times 2$ vertical torsion mirror switch.

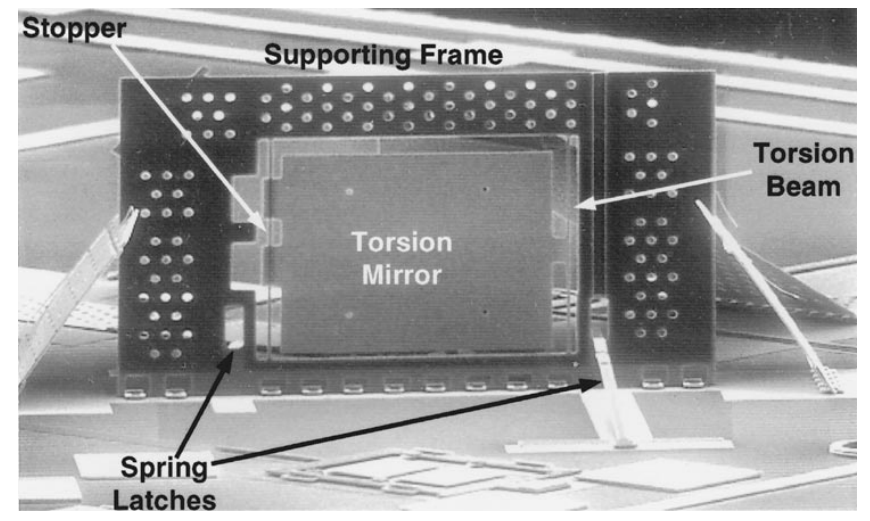

Fig. 4. Scanning electron micrograph (SEM) of the vertical torsion mirror.

minimize the separation between the assembled mirror and the back electrode. Fig. 5 illustrates the arrangement of the spring latches. The side support plates minimize the motion of the frame during switch operation. In addition, many large holes are etched on the frame to reduce the electrostatic attraction force between the back electrode and the frame.

\section{B. Back Electrode}

The back electrode consists of three polysilicon plates jointed together by microhinges: a back electrode plate, a 

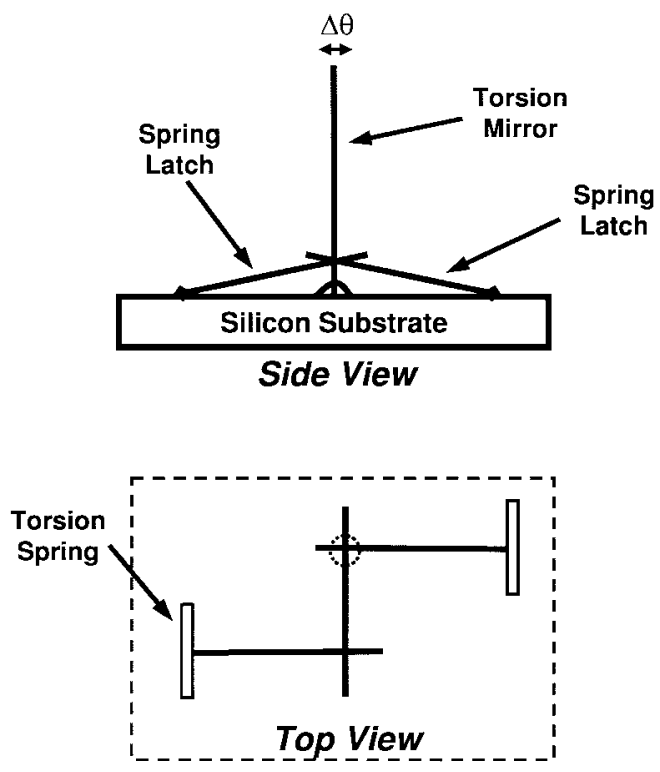

Fig. 5. Schematic drawing of the arrangement of two spring latches.

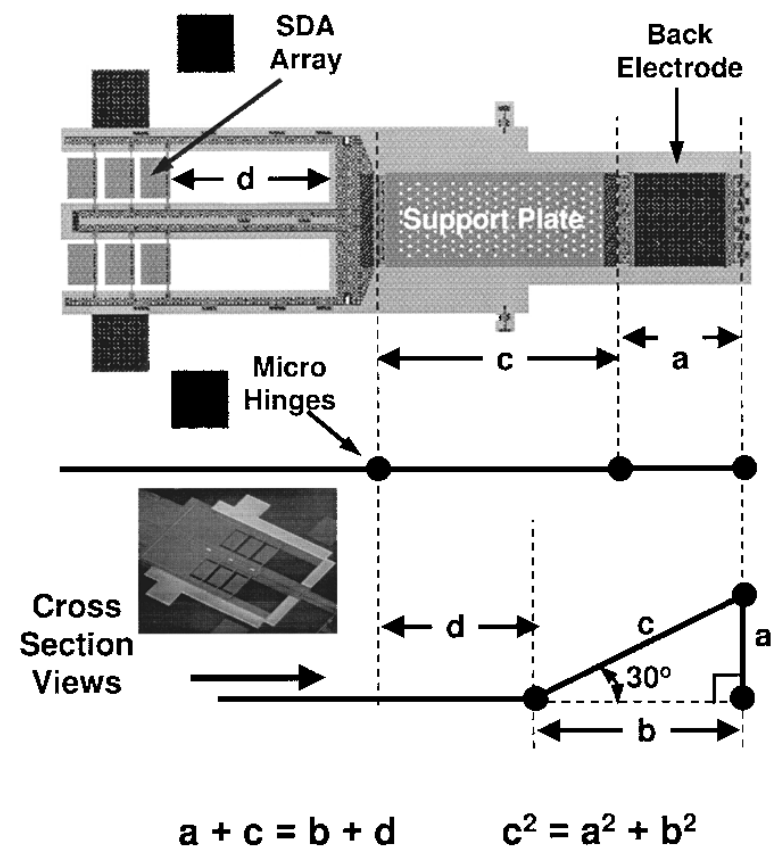

Fig. 6. Schematic illustration of self-assembly of the back electrode.

support plate, and an actuator plate for self-assembly as shown in Fig. 6. When a pulsed voltage is applied to the SDA arrays, the actuator plate moves toward the support plate, causing the microhinge between the support plate and the back electrode to buckle up. After assembly, the back electrode becomes perpendicular to the substrate. The geometry of the back electrode also enhances its mechanical strength, which is important to keep it stationary during switch operation. To prevent direct contact with the mirror during switch operation, a microstopper has been employed. The microstopper comprises another hinged vertical plate that is electrically connected to the torsion mirror plate. Since the microstopper is closer to the back electrode than the torsion mirror, the electrostatic
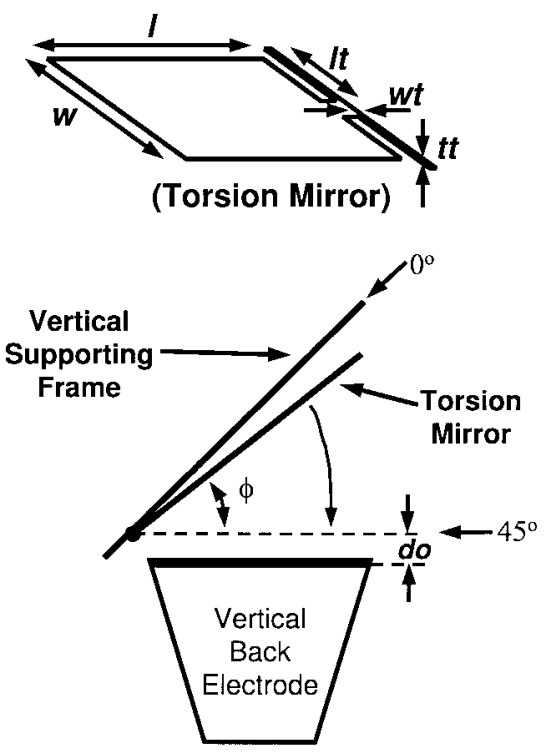

(Top View)

Fig. 7. Geometrical relation between the mirror and back electrode.

force between the micro-stopper and the back electrode must be taken into consideration. We have employed a triangular structure similar to the back electrode increase its mechanical strength and reduce the electrostatic force on the microstopper. The unassembled microstopper can be seen in the foreground of Fig. 2.

\section{Device Modeling}

The switch is designed to have a switching voltage less than $100 \mathrm{~V}$. Fig. 7 shows the geometrical relation between the mirror and the back electrode. The electrostatic torque $\left(T_{e}\right)$ exerted by the applied voltage (1) and the restoring torque $\left(T_{r}\right)$ of the torsion beam (2) can be expressed as functions of the rotation angle $\phi\left(\phi\right.$ varies from 0 to $\left.45^{\circ}\right)$ [2]

$$
\begin{aligned}
& T_{e}=\frac{\varepsilon \cdot w \cdot V^{2}}{2} \cdot \int_{0}^{l} \frac{x}{\left[\left(\frac{\pi}{4}-\phi\right) \cdot x+d_{0}\right]^{2}} d x \\
& T_{r}=\frac{2}{3} \cdot \frac{G \cdot w t \cdot t t^{3} \cdot \phi}{l t}\left(1-\frac{192}{\pi^{5}} \cdot \frac{t t}{w t} \cdot \tanh \left(\frac{\pi \cdot w t}{2 \cdot t t}\right)\right)
\end{aligned}
$$

where $w t, l t, t t$ are the width, length, and thickness of the torsion beam, respectively, $w$ and $l$ are the width and length of the mirror plate, respectively, $d_{0}$ is the separation between the torsion beam and the back electrode, $V$ is the applied voltage, $\varepsilon=8.85 \times 10^{-12} \mathrm{~F} / \mathrm{m}$ is the dielectric constant of the vacuum, and $G=73 \mathrm{GPa}$ is the shear modulus of polysilicon. Since the restoring torque is proportional to the cube of the torsion beam thickness, reducing the thickness is the most effective way to reduce the operating voltage without increasing the size of the switch. Here, the thickness of the torsion beam is reduced from $1.5 \mu \mathrm{m}$ to $0.55 \mu \mathrm{m}$ by reactive ion etching (RIE) with $\mathrm{SF}_{6}$ gas, which yields a pull-in voltage of around $80 \mathrm{~V}$. The calculated pull-in voltage versus the torsion beam thickness is shown in Fig. 8. In addition, optimizing the initial separation 


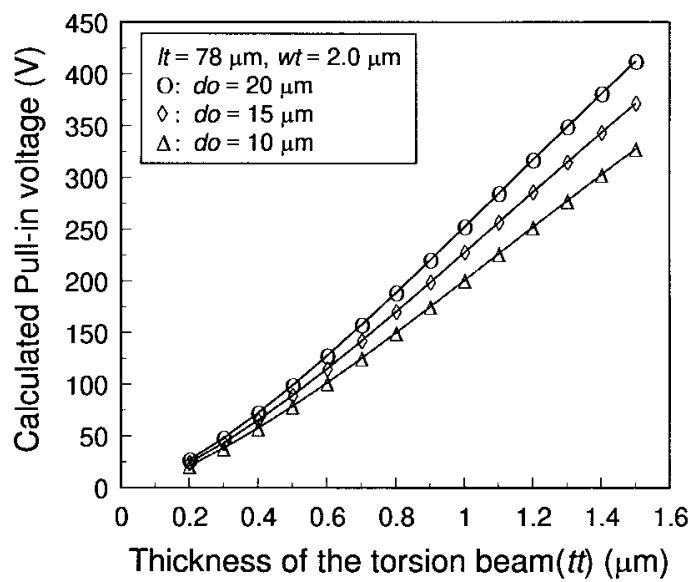

Fig. 8. Plot of torsion beam thickness versus calculated pull-in voltages.

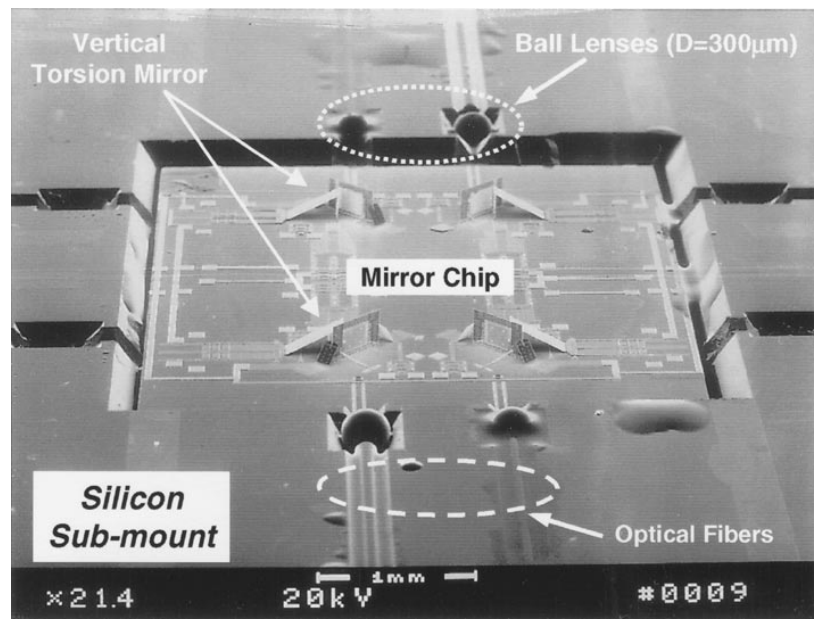

Fig. 9. Scanning electron micrograph (SEM) of the $2 \times 2$ fiber-optic switch.

$\left(d_{0}\right)$ between the torsion mirror and the back plate can further reduce the pull-in voltage. No change of the mechanical strength of the torsion mirror is observed after the etching.

\section{III. $2 \times 2$ Fiber Optic Switch ANd Slicon Submount}

The $2 \times 2$ fiber-optic switch consists of a mirror chip and a silicon submount. The SEM of the switch is shown in Fig. 9. The mirror chip comprising four surface-micromachined vertical torsion mirror devices is hybrid-integrated with the silicon submount. The principle of switch operation is illustrated in Fig. 10. The four vertical torsion mirror devices are arranged such that in the REFLECTION mode, the input beams are reflected by two $45^{\circ}$ vertical torsion mirrors and coupled into the output fibers located on the same side of the chip. In the TRANSMISSION mode, the vertical torsion mirrors are rotated out of the optical paths, and the input beams are coupled into the output fibers on the opposite side of the chip. The mirror chip is fabricated using the MUMP's process, while the submount is designed and fabricated at UCLA.

\section{A. Silicon Submount}

The bulk micromachined silicon submount is designed to achieve hybrid packaging of the switch with multimode
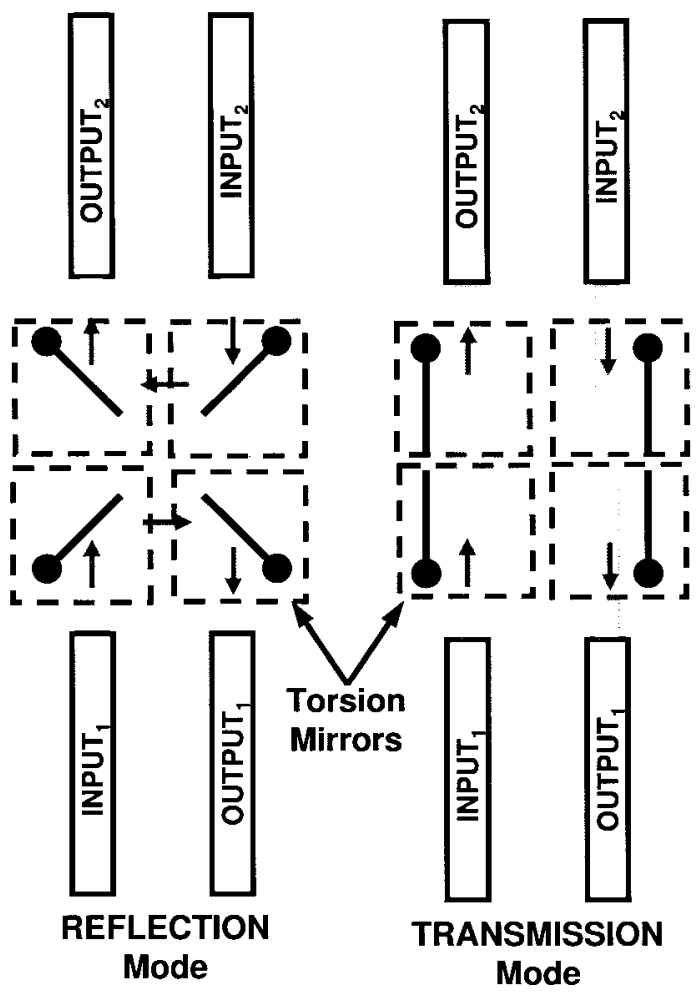

Fig. 10. Schematic illustration of operation principle of the $2 \times 2$ fiber-optic switch.

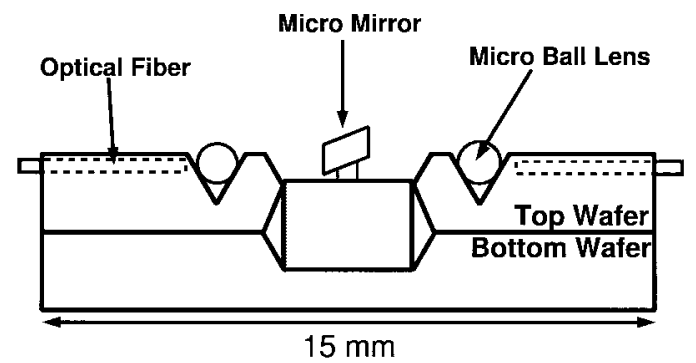

Fig. 11. Cross-section view of the package: a mirror chip placed on $\mathrm{Si}$ submount and covered.

fibers with passive optical alignment. Fig. 11 shows the crosssectional view of the packaged switch. The submount consists of two bulk-micromachined silicon wafers bonded together. The top wafer has a central opening whose area matches that of the mirror chip, four $\mathrm{V}$-grooves for aligning the optical fibers, and four micropits for holding the micro ball lenses. The bottom substrate has a central recess area and is bonded to the top substrate. With this hybrid packaging scheme, the mirror chip and the silicon submount can be fabricated and optimized separately, thus avoiding the complexity associated with fabricating all the features on a single chip.

Micro ball lenses are used to collimate the light from the optical fibers and increase the working distance. The depth of the $\mathrm{V}$-grooves and the micropits are designed to match the optical axes of the fibers and the micro ball lenses. The $\mathrm{V}$-grooves, micropits and central recess are realized by a single-step anisotropic etching of (100) silicon wafer. Corner compensation structures [13] are used to preserve the convex 


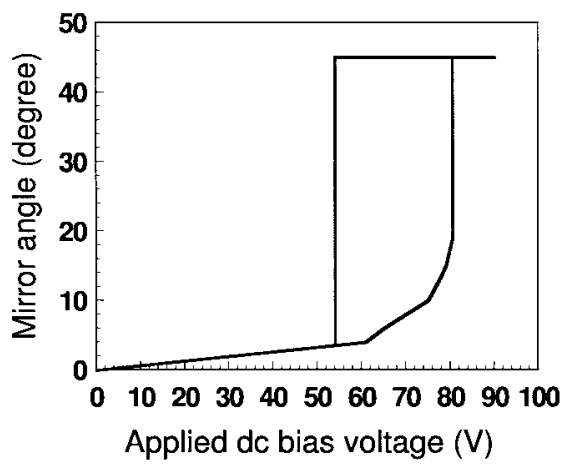

Fig. 12. DC switching characteristic.

corners during the etching process. Silicon wafers with (100) orientation are used for both the top (double polished) and the bottom substrates. The fabrication process is described in the following: first, a $1000-\AA$-thick silicon dioxide is thermally grown on the silicon wafer, followed by a 1500 - $\AA$-thick silicon nitride deposited by low-pressure chemical vapor deposition (LPCVD), which serves as the mask for anisotropic etching. With the help of the reference marks that are etched to reveal the true crystal direction, the edges of the V-groove patterns are aligned with the crystal orientation of the silicon wafer to within $0.1^{\circ}$ accuracy [14]. Both sides of the wafer are pattern by a double-sided mask aligner, and etch windows are opened by RIE with $\mathrm{CF}_{4} / \mathrm{O}_{2}$ plasma. Then the wafer is then etched in $\mathrm{KOH}$ until the central hole etches through. Finally, 5000 $\AA$-thick aluminum bonding pads are deposited by the lift-off process. The bottom silicon substrate is prepared by a simpler process to create the recess for the switch chip.

\section{B. Assembly}

The assembly process is described in the following: first, both wafers are diced into chips with areas of $15 \mathrm{~mm} \times 15$ $\mathrm{mm}$. The top and the bottom wafers are aligned by inserting dummy optical fibers into the matching V-grooves etched on the bonding surfaces of the wafers. The two mating wafers are bonded together by thermally cured adhesive with pressure applied. The dummy optical fibers are arranged in orthogonal directions to prevent translation or rotation of the wafers during the curing process. Then, the switch chip and the ball lenses are placed in the center opening and the micropits, respectively. Finally, four optical fibers are fixed in the Vgrooves by UV epoxy.

\section{EXPERIMENTAL RESULTS}

The dc switching characteristics is plotted in Fig. 12. The measured mirror angle versus the applied voltage curve exhibits a hysteresis that is characteristic of the electrostatic gap-closing actuators. A pull-in voltage of $80 \mathrm{~V}$ and a releasing voltage of $54 \mathrm{~V}$ have been achieved. The pull-in and releasing voltages can be further optimized by adjusting the thickness of the torsion beam, the area of the back electrode and the initial separation $\left(d_{0}\right)$ between the torsion mirror and the back plate. The switching time is characterized by monitoring the output light from Fiber B in Fig. 3 with an

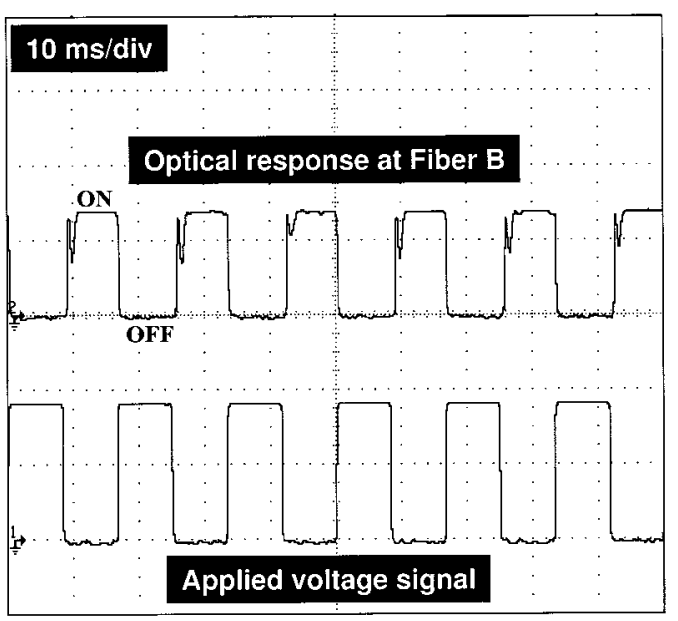

(a)

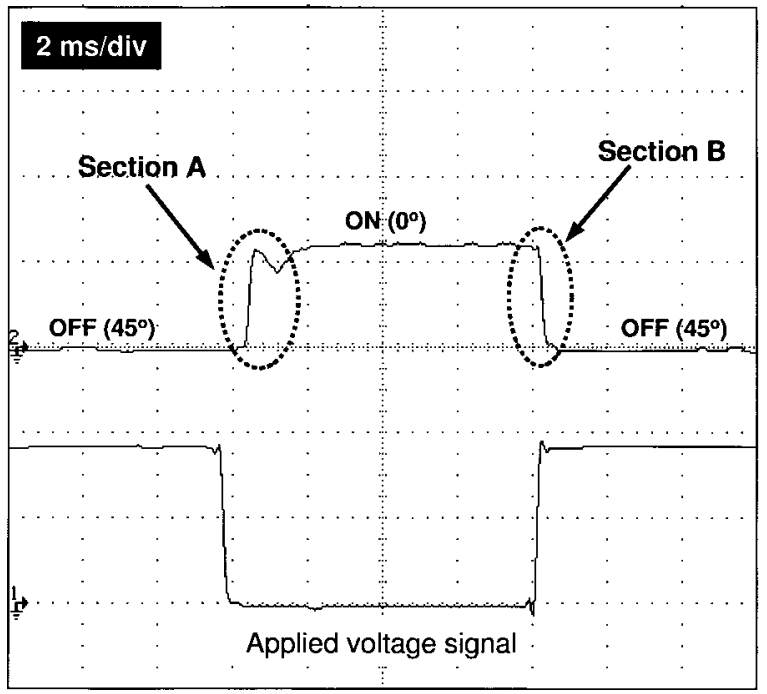

(b)

Fig. 13. (a) Dynamic response of the switch. (b) Section A: 45 -to- $0^{\circ}$ switching caused by the restoring torque of the torsion beam; Section B: 0-to- $45^{\circ}$ switching induced by the electrostatic torque.

oscilloscope. The result of dynamic switching is shown in Fig. 13(a). Section A [Fig. 13(b)] corresponds to the 45-to$0^{\circ}$ switching caused by the restoring torque of the torsion beam, and Section B [Fig. 13(b)] corresponds to the 0 -to- $45^{\circ}$ switching induced by the electrostatic torque. Switching time of less than $1 \mathrm{~ms}\left(t_{\text {rise }}=400 \mu \mathrm{sec}+t_{\text {delay }}=550 \mu \mathrm{sec}\right)$ and $400 \mu \mathrm{sec}$ have been measured for Sections A and B, respectively. We have observed some oscillations associated with the switching in Section A [Fig. 13(b)]. The oscillation is due to the mechanical impact as the mirror returns and collides with the micro-stopper on the frame. Using a programmable voltage signal can eliminate the oscillation due to the impact. The vertical torsion mirror can also be used as an optical scanner, the vertical torsion mirror has a stable scan range of $12^{\circ}$, which is sufficient for bar code scanners.

The optical insertion loss of the switch is characterized by three different optical setups. First, collimated beam fibers (CBF) from Namiki are used to measure the insertion loss of the $2 \times 2$ switch for single mode fibers. The CBFs are 
positioned by external XYZ stages with tilt control. A minimum optical insertion loss of $1.25 \mathrm{~dB}$ has been obtained for the $2 \times 2$ switch in the REFLECTION state. The losses include those from the two mirrors and the fiber coupling loss. For comparison, the insertion loss of the TRANSMISSION state is measured to be $0.55 \mathrm{~dB}$. The average loss per mirror deducted from the above data is $0.35 \mathrm{~dB}$. This includes the reflection loss, the scattering loss from the rough polysilicon surface, and the loss due to the slight curvature of the mirror. The crosstalk of the switch is below $-60 \mathrm{~dB}$ (measurement limit). We have also measured the insertion loss of the $1 \times 2$ switch with only one vertical torsion mirror. The insertion loss of the REFLECTION state is measured to be $0.9 \mathrm{~dB}$, which is consistent with data from the $2 \times 2$ switch.

The optical insertion loss for multimode fibers is characterized by both commercial multimode fiber collimators and the micro ball lenses on the silicon submount. Active optical alignment is employed for the commercial collimators. The insertion loss of the $1 \times 2$ switch is found to be $1 \mathrm{~dB}$ for a coupling distance of $600 \mu \mathrm{m}$. With passive optical alignment on the silicon submount, however, the optical insertion losses are found to be very high $(>10 \mathrm{~dB}$ ) for both the TRANSMISSION and the REFLECTION states. The primary loss is attributed to the residue angular misalignment caused by the variation of the ball lens diameters. Departure of the lens diameter from the designed value causes the input beam to tilt. This results in significant walk off of the optical beam when it reaches the output ball lens. The impact of angular misalignment can be minimized by reducing the working distance (the distance between the ball lenses). We estimate that an insertion loss of less than $2 \mathrm{~dB}$ can be achieved if the chip size is reduced to 1 $\mathrm{mm}$. Ball lenses with more accurate and uniform diameters will further improve the coupling loss. Though the loss is still higher than that obtained by active optical alignment, the silicon submount with passive alignment is attractive for low-cost fiber-optic switches.

\section{SUMMARY}

In conclusion, we have demonstrated a novel MEMS fiberoptic switch based on surface-micromachined vertical torsion mirrors. The switch is electrostatically driven and semi-selfassembled. The vertical torsion mirror can be used as a $1 \times 2$ optical switch, an ON-OFF switch, or an optical scanner. A $2 \times 2$ fiber-optic switch with multiple vertical torsion mirrors has also been fabricated. A switching voltage of $80 \mathrm{~V}$ and a switching time of less than $400 \mu$ s (fall time) have been achieved. Optical insertion loss of $1.25 \mathrm{~dB}$ and crosstalk of less than $-60 \mathrm{~dB}$ have been measured for the $2 \times 2$ switch with single-mode fibers.

\section{ACKNOWLEDGMENT}

The authors would like to thank Prof. H. Toshiyoshi of Tokyo University, Tokyo, Japan, for the loan of fiber collimators and Mr. H. Nguyen of the University of California, Los Angeles, for the measurement of the insertion loss with single-mode fibers.

\section{REFERENCES}

[1] M. F. Dautartas, A. M. Benzoni, Y. C. Chen, and G. E. Blonder, "A silicon-based moving-mirror optical switch," J. Lightwave Technol, , vol. 10, pp. 1078-1085, Aug. 1992.

[2] H. Toshiyoshi and H. Fujita, "Electrostatic micro torsion mirrors for an optical switch matrix," J. Microelectromechan. Syst., vol. 5, no. 4, pp. 231-237, Dec. 1996.

[3] C. Marser, C. Thio, M.-A. Gretillat, N. F. de Rooji et al, "Vertical mirrors fabricated by deep reactive ion etching for fiber-optic switching applications," J. Microelectromechan. Syst., vol. 6, no. 3, pp. 277-285, Sept. 1997.

[4] E. Ollier, P. Labeye, and F. A. Revol, "Micro-opto mechanical switch integrated on silicon," Electron. Lett., vol. 31, no. 23, pp. 2003-2005, Nov. 9, 1995.

[5] L. A. Field, D. L. Burriesci, P. R. Robrish, and R. C. Ruby, "Micromachined $1 \times 2$ optical-fiber switch," Sensors and Actuators A (Physical), vol. A53, no. 1-3, pp. 311-316, May 1996.

[6] L. Y. Lin, E. L. Goldstein, J. M. Simmona, and R. W. Tkach, "Freespace micromachined optical switches with submillisecond switching time for large-scale optical crossconnects," IEEE Photon. Technol. Lett., vol. 10, pp. 525-527, Apr. 1998.

[7] M. H. Kiang, O. Solgaard, K. Y. Lau, and R. S. Muller, "Electrostatic combdrive-actuated micromirrors for laser-beam scanning and positioning," J. Microelectromechan. Syst., vol. 7, no. 1, pp. 27-37, Mar. 1998.

[8] L. Y. Lin, S. S. Lee, K. S. J. Pister, and M. C. Wu, "Micro-machined three-dimensional micro-optics for integrated free-space optical system," IEEE Photon. Technol. Lett., vol. 6, Dec. 1994.

[9] S. S. Lee, L. Y. Lin, and M. C. Wu, "Surface-micromachined free-space micro-optical systems containing three-dimensional micro-gratings," Appl. Phys. Lett., vol. 67, no. 15, pp. 2135-2137, Oct. 1995.

[10] S. S. Lee and M. C. Wu, "Surface-micromachined free-space fiber optic switches with integrated microactuators for optical fiber communication," in Proc. Tech. Dig. 1997 Int. Solid-State Sensors and Actuators Conf.-TRANSDUCERS'97, Chicago, IL, June 16-19, 1997, vol. I, pp. $85-88$.

[11] K. S. J. Pister, M. W. Judy, S. R. Burgett, and R. S. Fearing, "Microfabricated hinges," Sensors and Actuators A (Physical), vol. 33, no. 3, p. 249, 1992.

[12] T. Akiyama, D. Collard, and H. Fujita, "Scratch drive actuator with mechanical links for self-assembly of three-dimensional MEMS," $J$. Microelectromechan. Syst., vol. 6, no. 1, pp. 10-17, Mar. 1997.

[13] L.-S. Huang, S. S. Lee, E. Motamedi, M. C. Wu, and C.-J. Kim, "MEMS packaging for micro mirror switch," in Proc. 48th IEEE Electron. Components Technol. Conf., Seattle, WA, May 25-28, 1998.

[14] G. Ensell, "Alignment of mask patterns to crystal orientation," Sensors and Actuators A (Physical), vol. A53, nos. 1-3, pp. 345-348, May 1996.

Shi-Sheng Lee (M'98) received the M.S. and Ph.D. degrees in electrical engineering from University of California, Los Angeles, in 1995 and 1998, respectively. His doctoral research involved investigation of MEMS free-space fiber optic switches.

His research interests include microfabrication, optical MEMS, integrated microoptical systems, microactuators, and microoptical source/detectors. From 1994 to 1996, he was a recipient of RAND/UCLA Fellowship. He is currently with AMP Inc. and conducting research in the MEMS fiber-optic components for telecommunication applications. He has authored and coauthored more than 40 technical papers.

Dr. Lee is a member of Eta Kappa Nu.

Long-Sun Huang, photograph and biography not available at the time of publication. 
Chang-Jin Kim received the B.S. degree from Seoul National University, Korea, and the M.S. degree from Iowa State University, Ames, with the Graduate Research Excellence Award. He received the Ph.D. degree in mechanical engineering from the University of California at Berkeley in 1991 with a study on MEMS.

$\mathrm{He}$ joined the faculty at the University of California at Los Angeles (UCLA) in Mechanical and Aerospace Engineering Department in 1993 after postdoctoral work at the University of California at Berkeley and the University of Tokyo, Japan. His research interest is in MEMS, including design and fabrication of microstructures, microactuators and systems, and use of surface tension in microscale. He has developed three graduate courses in MEMS at UCLA and is also active in various MEMS professional courses.

Dr. Kim is the recipient of the 1995 TRW Outstanding Young Teacher Award and the 1997 NSF CAREER Award. He served as Chairman of the Micromechanical Systems Panel of the ASME Dynamic Systems and Control Division in 1996 and since 1994, he has coorganized the Symposium on Micromechanical Systems for the ASME International Mechanical Engineering Congress and Exposition, for which he edited the Proceedings of MEMS in 1996 and 1997. He also organized the 1996 ASME Satellite Broadcast Program, MEMS: Case Studies of Commercial Products and served as General Co-Chairman of the 6th IEEE International Conference on Emerging Technologies and Factory Automation (ETFA'97). He is currently serving in the Technical Program Committees of the IEEE Micro-ElectroMechanical Systems Workshop and the SPIE Symposium on Micromachining and Microfabrication.
Ming C. Wu (S'82-M'88) received the M.S. and Ph.D. degrees in electrical engineering from the University of California, Berkeley, in 1985 and 1988, respectively.

From 1988 to 1992, he was Member of Technical Staff with AT\&T Bell Laboratories, Murray Hill, NJ, where he conducted research in highspeed semiconductor lasers and optoelectronics. In 1993, he joined the faculty of Electrical Engineering Department of University of California at Los Angeles (UCLA), and is currently a Professor. His current research interests include microelectromechanical systems, optical MEMS, free-space integrated optics, ultrafast integrated optoelectronics, microwave photonics, high-power photodetectors, semiconductor lasers, and optical interconnect. He has published over 95 journal papers, 160 conference papers, contributed one book chapter, and holds eight U.S. patents.

Dr. $\mathrm{Wu}$ is the Director of the MURI Center on RF Photonic Materials and Devices sponsored by ONR. He was General Co-Chair of the IEEE LEOS Summer Topical Meeting in 1995 (RF Optoelectronics), 1996 and 1998 (Optical MEMS), and Program Committee Chair of the 1997 International Conference on Optical MEMS. He has also served on the Program Committees of CLEO, OFC, IEDM, and DRC. He received the Packard Foundation Fellowship in 1992, and the Meritorious Conference Paper Award of 1994 GOMAC. He is a member of the American Physical Society, Optical Society of America (OSA), URSI, and Eta Kappa Nu. 